\title{
Co-factor influences the severity of Zika virus-associated microcephaly
}

Adriano B. Nogueira ${ }^{a, b^{*}}$, Breno B. Andrade ${ }^{c}$, Leonardo Y. K. Zaninic ${ }^{\text {, Hillary S. R. Hoshino }}{ }^{\mathrm{c}}$, Natalia C. Ortega ${ }^{c}$, Adriana Mattos ${ }^{\mathrm{d}}$, Ana Paula A. P. Bertozzi ${ }^{\mathrm{e}}$, Manoel J. Teixeira ${ }^{\mathrm{a}, \mathrm{f}}$

${ }^{a}$ Division of Neurosurgery, Hospital das Clínicas, Faculty of Medicine, University of São Paulo, São Paulo, Brazil

${ }^{\mathrm{b}}$ Neurosurgery Service, Hospital Regional do Vale do Paraíba, Taubaté, Brazil

${ }^{c}$ Department of Medicine, University of Taubaté, Taubaté, Brazil

${ }^{\mathrm{d}}$ Hospital Geral Roberto Santos, Secretaria da Saúde do Estado da Bahia, Salvador, Brazil

${ }^{\mathrm{e}}$ Independent Researcher

${ }^{\mathrm{f}}$ Department of Neurology, Faculty of Medicine, University of São Paulo, São Paulo, Brazil

* corresponding author: adriano.nogueira@hc.fm.usp.br 


\begin{abstract}
Microcephaly has been regarded the most remarkable consequence of the Zika virus (ZIKV) epidemic in Brazil 2015. It remains to be determined whether there are factors that contribute to the degree of brain lesion associated with ZIKV infection during pregnancy. Previous studies showed that socioeconomic conditions correlate with ZIKV-associated microcephaly. Certain nutritional deficits display the potential to interfere in the mechanistic target of rapamycin (mTOR) signaling, which plays a major role in the pathophysiology of ZIKV-associated microcephaly. We hypothesize that a nutritional or environmental co-factor that interferes in mTOR signaling correlates with ZIKVassociated birth defects. To assess this hypothesis, we plan to: 1) develop a mouse model of ZIKVassociated microcephaly through intravenous injection of ZIKV and rapamycin for a straightforward interference on mTOR receptor; 2) determine in the experimental model and in cases of ZIKVassociated microcephaly the epigenetic signature (DNA methylation pattern) in neurons and muscle cells harvested by biopsy, and in hematopoietic and mesenchymal stem cells sorted from blood; 3 ) analyze through mass spectrometry in serum of pregnant female mice submitted to ZIKV and rapamycin injection and in serum of mothers of children with ZIKV-associated microcephaly the metabolomic pattern of cholesterol (a nutritional status marker), vitamin A and its metabolite retinoic acid, folate, and other metabolites related to these three nutritional factors; 4) check whether pregnant female mice submitted to intravenous injection of ZIKV and feed with a deficient diet of the most likely co-factor found in this study give birth to microcephalic mice with features that mimic clinical cases. In summary, our general objective is to develop an experimental model that mimics ZIKVassociated microcephaly cases and to find a co-factor involved in the microcephaly outbreak in Brazil 2015.
\end{abstract}

Keywords: Zika virus, Microcephaly, Neurogenesis, Retinoic Acid, mTOR signaling 


\section{Introduction}

In Brazil 2015, a microcephaly outbreak coincided with a Zika virus (ZIKV) epidemic. The detection of ZIKV infection in microcephalic children and in their mothers during pregnancy led to the conclusion that ZIKV was the cause of microcephaly [1]. Further observations detected a spectrum of ZIKV-associated brain alterations, in which microcephaly is the most severe pathological feature [1]. The reasons for which ZIKV infection leads to different degrees of brain lesions are not totally clear. An important epidemiological observation in this regard is that $\mathrm{ZIKV}$-associated microcephaly is more common in populations with poor socioeconomic background [2].

It is possible that a poor socioeconomic background correlates with the degree of severity of ZIKVassociated brain lesions due to nutritional or environment features [3]. Populations with higher incidence of ZIKV-associated microcephaly display higher incidence of malnutrition, vitamin A deficit [4,5], and folate deficit during pregnancy, for example [6]. Through different mechanisms, these nutritional alterations can lead to alterations in the placental barrier, fetal blood-brain barrier, and neurogenesis $[7,8]$. Therefore, certain nutritional deficits could facilitate the invasion of the ZIKV into the placenta and the fetal brain and amplify the presence of the virus in the fetus.

These nutritional factors act through different mechanisms that converge to the common set of molecules that are part of the insulin like growth factor (IGF)-1 / insulin and mechanistic target of rapamycin (mTOR) signaling pathway [9-12]. MTOR signaling pathway has been implicated as the most affected signaling in ZIKV-associated microcephaly [13]. Malnutrition interferes in glucose metabolism and insulin level and action [15]. Insulin acts on IGF-1 receptor - which displays a major role in neurogenesis [13] - and sequentially in mTOR signaling [15]. Vitamin A is the primary source of retinoic acid, which is involved in neurogenesis directly and indirectly via modifications in the expression of IGF-1 [8,9,16-18]. Folate is a known factor for normal neural tube formation and the decrease of its level leads to a decrease in mTOR [11]. Therefore, nutritional alterations in geographic zones with ZIKV-associated microcephaly could lead to alterations in the IGF-1 / insulin and mTOR signaling pathway. These alterations could influence the degree of ZIKV-associated brain lesions.

ZIKV infection has been associated with birth defects in experimental models, but certain points remain unexplained. A study reported a nonhuman primate submitted to subcutaneous ZIKV injection during pregnancy, followed by the development of malformations in the fetus brain [19]. The pathologic analysis of this brain revealed the presence of ZIKV-associated findings. Nonetheless, this study analyzed only one animal and the injected viral load was high. Moreover, another nonhuman primate model failed to provoke ZIKV-associated brain malformations [19]. ZIKV-associated brain malformations in mice have been shown only through direct intracerebral injection of the virus in the fetus or in fetus of strains with alterations such as early tumor development or immunodeficiency [20]. In vitro studies with neural stem cells, neurospheres, and cerebral organoids revealed ZIKV capacity to infect and damage these structures [20]. Nonetheless, these in vitro methods do not allow conclusions regarding the role of protection against fetal ZIKV infection by the placental and bloodbrain barriers.

In short, an experimental model that proves that ZIKV infection alone causes microcephaly is missing $[19,20]$. For this reason, we hypothesize that a nutritional or environmental factor contributes to ZIKVassociated microcephaly. The discovery of such factor contributing to the occurrence of microcephaly in ZIKV cases could lead to the development of an experimental model that mimics the real clinical condition. This model could underpin the discovery of mechanisms of the disease and lead to therapeutic measures. 
The sharp drop in the incidence of cases of ZIKV-associated microcephaly hampers the comparison between an experimental model and cases with recent disease installation. The comparison should be performed with a parameter that remains in the animal model until the counterpart age of the children with ZIKV-associated microcephaly born during the ZIKV epidemic in Brazil. This epidemic occurred six years ago. One strategy to tackle this obstacle is to compare in children with or without ZIKVassociated microcephaly the DNA methylation pattern developed during fetal formation that remains in infanthood [21-23]. This epigenetic pattern in microcephalic children would serve to validate the experimental model and to reveal a signature of ZIKV-associated microcephaly potentially useful for diagnosis, prognosis, and therapy purposes. Likewise, a metabolomic profile [24] of the serum of mothers of microcephalic children could in theory help to validate the experimental model, if this metabolomic profile is similar to the profile found in pregnant female mice of the experimental model. A caveat regarding this assumption is that the metabolomic profile could have changed since the pregnancy of a child with microcephaly due to changes in nutritional profile, for example.

\section{Hypotheses}

We hypothesize that ZIKV-associated microcephaly displays an environmental co-factor that influences the degree of severity of the disease. We also hypothesize that ZIKV cannot cross integral placental and the fetal blood-brain barriers sufficiently to lead to microcephaly formation in humans and experimental models.

\section{Specific aims to test the hypotheses}

Aim \#1

To develop an experimental model of microcephaly by intravenous injection of the American strain of ZIKV and rapamycin in pregnant female mice. Rapamycin causes brain defects [25] and acts on the most affected signaling pathway in ZIKV-associated microcephaly, namely, the mTOR signaling [13].

\section{Aim \#2}

To compare the epigenetic signature (DNA methylation pattern) of neurons, muscle cells, hematopoietic and mesenchymal stem cells in the experimental model and in children with ZIKVassociated microcephaly.

\section{Aim \#3}

To compare the metabolomic pattern of the serum of the pregnant female mice with the pattern of the serum of mothers of children with ZIKV-associated microcephaly using mass spectrometry. The focus will be on potential nutritional co-factors, namely, cholesterol, vitamin A / retinoic acid, and folate and their metabolites.

\section{$\operatorname{Aim} \# 4$}

To verify whether pregnant female mice submitted to intravenous injection of ZIKV and a diet with deficiency of the most likely co-factor for ZIKV-associated microcephaly (or an exposure to a likely environmental co-factor) generates a microcephalic offspring. In addition, to assess whether the microcephalic mice display epigenetic, metabolomic, and radiologic patterns similar to those found in children with ZIKV-associated microcephaly. 


\section{Evaluation of the hypotheses}

\section{Development of the experimental model}

The mechanisms of potential nutritional co-factors of ZIKV-associated microcephaly converge to the IGF-1 / insulin and mTOR signaling, which is strongly involved in neurogenesis and in the pathogenesis of ZIKV-associated microcephaly [13]. Because of these features, the first approach of this project will be pursuing the development of a ZIKV-associated microcephaly model via the intravenous injection of ZIKV and rapamycin in pregnant female mice.

One mouse in the first third of gestation will receive intravenous injections of rapamycin described as sufficient to yield viable microcephalic offspring [25] and injection of ZIKV proportional to the viral burden estimated for mosquito bite in infection of humans [19]. For comparison, one pregnant female mouse will receive no intervention, one will receive rapamycin injection only, and one will receive ZIKV injection only. All fetuses (including the negative control) will be submitted to brain magnetic resonance imaging (MRI) one day after the injection of rapamycin and ZIKV, at the end of the second third of gestation, and one day after birth. Regarding this imaging approach, the comparison among the groups will be qualitative. In the second day of life, the mice will be sacrificed, their brains will be weighted and processed for immunohistochemistry and transmission electron microscope analysis. The ratio between brain and body weights will be compared among the groups using the Mann-Whitney U test. Brain, temporal muscle, and blood samples will be separated for further epigenetic study through DNA methylation analysis [26]. This set of experiments will be repeated twice, with occasional modifications in the rapamycin dose or ZIKV viral burden if necessary.

In the end of this phase of the project we expect to have three pregnant female mice that generated offspring with brain malformation after the injection of ZIKV and rapamycin during the first third of pregnancy. We will carry out histology, immunohistochemistry, and electron microscopy experiments to seek for microscopic alterations and ZIKV invasion into the brain. Brain samples will be fixed in formalin and embedded in paraffin. Hematoxylin-eosin staining will be carried out following the protocol we have already used [27]. We plan to use a Nissl staining protocol available on the Internet [28]. Chromogenic immunohistochemistry will be performed using ImmPRESS ${ }^{\mathrm{TM}}$ Duet Double Staining HRP/AP Polymer Kit, Peroxidase (catalog no. MP7724) (Maravai Life Sciences, Vector Laboratories, San Diego, CA, USA), following the manufacture's recommendations. Fluorescence immunohistochemistry protocol will be same as we have previously detailed (see Additional File 1: Table S1: Fluorescence immunohistochemistry protocol in ref. 27). The microscopes to be used for slide analyses will be those used in our previous work (see Table 2 of ref. 27). Harvesting, processing, and image acquisition regarding electron microscopy will have the assistance of the multiuser facilities and services of the University of Sao Paulo Faculty of Medicine ("rede Premium" [29]). The electron microscope that will be used is JEOL JEM 1010 (Jeol Ltd., Tokyo, Japan) [29].

\section{Microscopic analysis of the experimental model}

The brain zones chosen for microscopic analysis are the neocortex, head of the caudate nucleus, dentate gyrus of the hippocampus, and olfactory bulb. These zones are representative of ZIKV-associated brain lesions or zones in which neurogenesis persists even after birth [27].

Hematoxylin-eosin and Nissl staining will be the histologic methods used to quantify neurons. Regarding immunohistochemistry, the markers used will be nestin for neural stem cells, doublecortin (DCX) and PSA-NCAM for immature neurons, and NeuN for mature neurons [27]. Neurons and cells expressing each of the neurogenesis-related markers will be quantified in terms of number per $\mathrm{mm}^{2}$ in 
each of the zones mentioned above. We will follow the approach we used previously to quantify DCX+ cells in the human hypothalamus in each of the zones mentioned above (see Fig. 17 in ref. 27). This density of cells will be compared among the groups using the Mann-Whitney U test. The test will be performed for each of the cell types in each of the analyzed brain zones. We expect to find at least a decrease in the expression of one marker in one zone in the ZIKV + rapamycin model in comparison to the negative control.

Electron microscopy experiments will be performed following standardized protocol, as mentioned above. The expected result is the detection of ZIKV in brain cells and morphological alterations in neurons only in the ZIKV + rapamycin model.

In short, in this phase of the project the expected result is the discovery of a correlation between gross and microscopic brain alterations in the ZIKV + rapamycin model. If we fail to find gross anatomy alterations, we expect to at least detect ZIKV in the brain with or without microscopic alterations, showing that the model serves for the virus to bypass the placental and fetal blood-brain barriers.

Search of signature of epigenetic alterations in experimental model and clinical cases of ZIKVassociated microcephaly

The main idea behind the design of the experimental model is that it aims at figuring out an animal model that mimics the role of co-factors, mechanisms, and clinical features of ZIKV-associated microcephaly. Because new cases of ZIKV-associated microcephaly dropped practically to zero for an unknown reason, the animal model should provide information comparable with children currently with six years of age who developed ZIKV-associated microcephaly during the 2015 ZIKV epidemic in Brazil.

A manner to seek this goal is via the analysis of DNA methylation pattern that could have remained for six years [21-23]. Accordingly, embryonic formation starts with no DNA methylation and progresses with cumulative and stable epigenetic modifications conveyed from progenitor cells to their progeny. In this manner, cells with low rate of division after birth are suitable candidates for cells that preserve the same overall pattern of these chromatin modifications in the long-term. Therefore, the action of ZIKV and a co-factor on the fetus could lead to a specific epigenetic pattern in certain cells that would remain practically unaltered in the long-term [26]. In practice, such epigenetic pattern could be sought with the use of the high throughput bisulfite sequencing analysis together with bioinformatics tools.

In the experimental model, bisulfite sequencing will be carried out using neurons, muscle cells, and hematopoietic and mesenchymal stem cells, which can be harvested from circulating blood using flow cytometry [21-23]. Neurons and muscle cells display no division after birth and are cells less prone to epigenetic modifications [26]. Mesenchymal and hematopoietic stem cells are non-differentiated types of cells more accessible in humans (they can be obtained with blood collection, with no need for biopsy) [21-23]. These cell types will be obtained in the first day of life and after 30 days from three mice of any of the four groups (control, ZIKV + rapamycin, ZIKV, and rapamycin). Complementary, these mice will be investigated with MRI in the $31^{\text {st }}$ day of life and next sacrificed; their brains will be processed for the same uses as the mice brains obtained after the first day of life. Using bisulfite sequencing and bioinformatics tools, we expect to find a specific epigenetic signature in neurons, muscle cells, and mesenchymal and hematopoietic stem cells harvested at days 1 and 30 of life in the ZIKV + rapamycin model. 
In microcephalic children, neurons [30] and muscle cells [31] will be obtained from patients submitted to neurosurgical procedures, principally ventriculoperitoneal shunt placement or revision [32]. Cortex samples of about 1-2 mL will be collected during the corticotomy usually performed as part of these surgical procedures. These samples will be processed according to the protocol of primary culture of human neurons detailed by Park et al. [30] Muscle sample of about $10 \mathrm{~mm}^{3}$ can be obtained from occipital or abdominal muscles, depending on the type of shunt surgery performed. Human skeletal muscle cells will be selected using the protocol detailed by Soriano-Arroquia et al. [31] We will collect neurons and muscle cells from three children.

Mesenchymal [33] and hematopoietic [34] stem cells will be obtained from nine children with indication of blood panel exam. Mesenchymal stem cells will be obtained following the protocol proposed by Ouryazdanpanah et al. [33] The advantages of this protocol are that it uses a small amount of human peripheral blood sample, avoid the necessity of mobilization with granulocyte-colony stimulating factor (G-CSF), and yields a large number of mesenchymal stem cells. These cells will be selected using cell sorting (BD FACS Aria II Flow Cytometry Cell Sorter (Becton Dickinson, Franklin Lakes, USA)). Cells regarded mesenchymal stem cells will be those expressing CD90, CD105, and CD73 and not expressing CD45 [33]. Similarly, hematopoietic stem cells will be selected using the method reported by Lemos et al. [34] to quantify CD34+ cells. These CD34+ cells will be obtained from non-mobilized peripheral blood, which is sufficient to carry out the epigenomics analyses of this project [35].

Controls of neurons and muscle cells will be obtained during brain surgery of children with comparable ages and with traumatic brain injury. Control of mesenchymal and hematopoietic stem cells will be obtained from children with indication of blood panel analysis whose results were normal. The number of samples for neurons, muscle cells, and mesenchymal and hematopoietic stem cells in the control group will be the same as for the microcephaly group. These cell types will be analyzed by bisulfite sequencing and bioinformatics tools following the same protocol used for the experimental model. We will match the epigenetic pattern of the cells from microcephalic children with the human controls and with the experimental model of ZIKV-associated microcephaly developed in this study. The rationale for this approach is that the DNA methylation pattern overlaps significantly between rodents and humans. To increase the change to find this overlap, we intend to take a closer look to transcription factor binding sites [36]. Recently, Zhou et al. [36] demonstrated the potential paradigm shift in Comparative Epigenomics using this type of analysis. Accordingly, the modification in the DNA methylation pattern by environmental stressors may be similar in different species [37]. The expected result is that the epigenetic signature in cells of microcephalic children is significantly similar to the pattern observed in the ZIKV + rapamycin model to be developed in this study. This result would suggest that the severity of ZIKV-associated brain malformations correlates with a (potentially reversible) co-factor that disturbs the IGF-1 / insulin and mTOR signaling.

\section{“Reverse engineering” to reveal co-factor of ZIKV-associated microcephaly}

At this phase of the project, the results are expected to suggest that a co-factor in ZIKV-associated microcephaly leads to an epigenetic signature in microcephalic children that remains until infanthood. This co-factor should act via a mechanism strongly involved in neurogenesis, i.e., the IGF-1 / insulin and mTOR signaling. The interference in this signaling and ZIKV infection would generate an animal model that mimics the cause, role of co-factors, mechanisms, and pathology of ZIKV-associated microcephaly. Pieces of evidence accumulated at this point of the project could guide the discovery of the co-factor itself. 
Accordingly, a putative co-factor in ZIKV-associated microcephaly could be suspected using epidemiological and experimental data, the results of previous phases of this project, and public repositories of epigenomics and metabolomics as explained below.

Epidemiological data search shows that ZIKV-associated microcephaly is more frequent in areas with poor socioeconomic background with reports of malnutrition and deficits of vitamin A [4,5] and folate [6]. A screening based on these epidemiological data will be performed through common exams for cholesterol and fractions (as a marker of nutritional status) [38], vitamin A, and folate in blood of nine mothers of microcephalic children. Metabolites associated with these small molecules will be analyzed using mass spectrometry. For comparison, the same experiments with mass spectrometry will be carried out with nine healthy adult women and with all pregnant female mice at the middle of gestation. We expect that the simultaneous action of rapamycin and ZIKV leads to a metabolomic profile that is similar to the metabolomic profile found in mothers of microcephalic children. The metabolomic profile will be focused on cholesterol, vitamin A / retinoic acid, and folate and their related metabolites.

To figure out if one of the suspected co-factors is a culprit of the increase of severity of ZIKVassociated macrocephaly, we will consider three points. These points are the screening with blood samples of the mothers, the epigenetic patterns found in the experimental model and in the cells of microcephalic children, and public repositories on epigenomics and metabolomics. Alternatively, another more likely co-factor may be revealed by our bisulfite sequencing and mass spectrometry experiments and epigenomics and metabolomics databases. The most likely co-factor will be tested in pregnant female mice submitted to intravenous injection of ZIKV in a more straightforward manner. For example, depending on previous results, we may opt to feed the pregnant female mice with hypocaloric, vitamin A deficient or folate deficient diet during pregnancy. We opted for not using all of these alternatives since the beginning because we want to assess the mechanisms of ZIKV-associated microcephaly. Furthermore, we do not rule out that, indeed, the previous steps of the project enhance indications regarding the role of another previously suspected co-factor that interferes in retinoic acid [39-41]. In other words, the study design proposed here took into account the most likely strategy to succeed in the test of the hypothesis that ZIKV infection alone does not explain totally the fetal brain malformation, which is the most common situation in clinical practice.

In this manner, the final phase of the project consists in performing the same experiments as in the first phase, but having as groups a negative control, low co-factor diet plus ZIKV injection, low co-factor diet only, and ZIKV injection only. MRI, histology, immunohistochemistry, and electron microscopy will be performed as in the first phase. Three groups of four pregnant female mice will be tested, and mice will be sacrificed with 30 days of life.

\section{Feasibility}

Children with ZIKV-associated microcephaly will be enrolled at Hospital Geral Roberto Santos, Salvador, Brazil, where approximately 100 cases have been followed. Project execution will also be carried out at University of São Paulo, University of Taubaté, and Hospital Regional do Vale do Paraíba, Taubaté, Brazil. The team includes researchers with expertise in medical neurosciences, neurogenesis, imaging, ZIKV, and molecular biology research.

\section{Consequences of the Hypothesis}

The expected result is that these experiments yield an animal model that mimics the radiological, pathological, and epigenetic features observed in children with ZIKV-associated microcephaly. This result would suggest that a co-factor amplifies the ZIKV effects on fetus brain. The prospects are that 
this co-factor can be corrected in the population of epidemic zones of ZIKV to at least mitigate the ZIKV-associated brain lesions.

\section{Conflict of Interest Statement}

The authors declare that the research was conducted in the absence of any commercial or financial relationships that could be construed as a potential conflict of interest.

\section{Consent Statement / Ethical Approval}

Not required

Funding

None

\section{Acknowledgments}

The authors thank to students of Neurology and Neurosurgery Interest Group, Department of Medicine, University of Taubaté, Taubaté, Brazil

\section{References}

1. Araújo TVB, Ximenes RAA, Miranda-Filho DB, et al. Association between microcephaly, zika virus infection, and other risk factors in Brazil: final report of a case-control study. Lancet Infec Dis 2018;18:328-36.

2. Butler D. Brazil asks whether Zika virus acts alone to cause birth defects. Nature 2016;535:475-6.

3. Pedrosa CSG, Souza LRQ, Gomes TA, et al. The cyanobacterial saxitoxin exacerbates neural cell death and brain malformations induced by Zika virus. PLoS Negl Trop Dis 2020;14: e0008060. https://doi.org/10.1371/journal.pntd.0008060.

4. Novaes TG, Gomes AT, Silveira KC, et al. Prevalence and factors associated to vitamin A deficiency in children attending public day care centers in the Southwest of Bahia. Rev Bras Saude Mater Infant 2016;16:337-44.

5. Maia SB, Caminha MFC, da Silva SL, Souza ASR, dos Santos CC, Batista Filho M. The prevalence of vitamin A deficiency and associated factors in pregnant women receiving prenatal care at a reference maternity hospital in Northeastern Brazil. Nutrients 2018:10:1271. https://doi.org/10.3390/nu10091271.

6. Palchetti CZ, Steluti J, Verly Jr E, Pereira RA, Sichieri R, Marchioni DML. Prevalence of inadequate intake of folate after mandatory fortification: results from the first National Dietary Survey in Brazil. Eur J Nutr 2020;59:2793-803.

7. Rhinn M., Dollé P. Retinoic acid signalling during development. Development 2012;139:84358.

8. Mate A, Reyes-Goya C, Santana-Garrido A, Sobrevia L, Vázquez CM. Impact of maternal nutrition in viral infections during pregnancy. Biochim Biophys Acta Mol Basis Dis 2021;1867:166231. https://doi.org/10.1016/j.bbadis.2021.166231.

9. Das BC, Thapa P, Karki R, et al. Retinoic acid signaling pathways in development and diseases. Bioorg Med Chem 2014;22:673-83.

10. Cunningham TH, Duester G. Mechanisms of retinoic acid signalling and its roles in organ and limb development. Nat Rev Mol Cell Biol 2015;16:110-23. 
11. Rosario FJ, Nathanielsz PW, Powell TL, Jansson T. Maternal folate deficiency causes inhibition of mTOR signaling, down-regulation of placental amino acid transporters and fetal growth restriction in mice. Sci Rep 2017;7:3982. https://doi.org/10.1038/s41598-017-03888-2.

12. Weissleder C, Barry G, Fung SJ, et al. Reduction in IGF-1 mRNA in the human subependymal zone during aging. Aging Dis 2019;10:197-204.

13. Caires-Júnior LC, Goulart E, Melo US, et al. Discordant congenital Zika syndrome twins show differential in vitro viral susceptibility of neural progenitor cells. Nat Comun 2018;9:475. https://doi.org/10.1038/s41467-017-02790-9.

14. Ozanne S, Hales C. The long-term consequences of intra-uterine protein malnutrition for glucose metabolism. Proc Nutr Soc 1999;58,615-9.

15. Saxton RA, Sabatini DM. MTOR signaling in growth, metabolism, and disease. Cell 2017;169:361-71.

16. Maia SB, Souza ASR, Caminha MFC et al. Vitamin A and pregnancy: a narrative review. Nutrients 2019;11:681. https://doi.org/10.3390/nu11030681.

17. Haushalter C, Asselin L, Fraulob V, Dollé P, Rhinn M. Retinoic acid controls early neurogenesis in the developing mouse cerebral cortex. Dev Biol 2017;430:129-41.

18. Lewitt MS, Boyd GW. The role of insulin-like growth factors and insulin-like growth factorbinding proteins in the nervous system. Biochem Insights 2019;12:1178626419842176. https://doi.org/10.1177/1178626419842176.

19. Waldorf KMA, Stencel-Baerenwald JE, Kapur RP, et al. Fetal brain lesions after subcutaneous inoculation of Zika virus in a pregnant nonhuman primate. Nat Med 2016;22:1256-9.

20. Hickman HD, Pierson TC. Zika in the brain: new models shed light on viral infection. Trends Mol Med 2016;22,639-41.

21. Ladd-Acosta C. Epigenetic signatures as biomarkers of exposure. Curr Environ Health Rep 2015;2:117-25.

22. Kim M, Costello J. DNA methylation: an epigenetic mark of cellular memory. Exp Mol Med 2017;49:e322. https://doi.org/10.1038/emm.2017.10.

23. Machnik M, Oleksiewicz U. Dynamic signatures of the epigenome: friend or foe? Cells 2020;9:653. https://doi.org/10.3390/cells9030653.

24. Markley JL, Brüschweiler R, Edison AS, et al. The future of NMR-based metabolomics. Curr Opin Biotechnol 2017;43:34-40.

25. Lu Z, Liu F, Chen L, et al. Effect of chronic administration of low dose rapamycin on development and immunity in young rats. PLoS One 2015;10:e0135256. https://doi.org/10.1371/journal.pone.0135256.

26. Janssens S, Schotsaert M, Karnik R, et al. Zika virus alters DNA methylation of neural genes in an organoid model of the developing human brain. MSystems 2018;3:1-12.

27. Nogueira AB, Sogayar MC, Colquhoun A, et al. Existence of a potential neurogenic system in the adult human brain. J Transl Med 2014;12:75. https://doi.org/10.1186/1479-5876-12-75.

28. http://www.ihcworld.com/_protocols/special_stains/nissl.htm (Accessed Sep 8, 2021).

29. https://www.premium.fm.usp.br/index.php?mpg=11.10.02\&lab=ME\&equipo=26 (Accessed Aug 22, 2021).

30. Park TI, Schweder P, Lee K, et al. Isolation and culture of functional adult human neurons from neurosurgical brain specimens. Brain Commun 2020;2:fcaa171. http://doi.org/10.1093/braincomms/fcaa171.

31. Sorriano-Arroquia A, Clegg PD, Molloy AP, Goljanek-Whysall K. Preparation and culture of myogenic precursor cells/primary myoblasts from skeletal muscle of adult and aged humas. $\mathrm{J}$ Vis Exp 2017;16:55047. http://doi.org/10.3791/55047.

32. Jucá E, Pessoa A, Ribeiro E, et al. Hydrocephalus associated to congenital Zika syndrome: does shunting improve clinical feature? Chids Nerv Syst 2018;34:101-6. 
33. Ouryazdanpanah N, Dabiri S, Derakhshani A, Vahidi R, Farsinejad A. Peripheral bloodderived mesenchymal stem cells: growth factor-free isolation, molecular characterization and differentiation. Iran J Pathol 2018;13:461-6.

34. Lemos NE, Farias MG, Kubaski F, et al. Quantification of peripheral blood CD $34^{+}$cells prior to stem cell harvesting by leukapheresis: a single center study. Hematol Transfus Cell Ther 2018;40:213-8.

35. Greenleaf WJ. Assaying the epigenome in limited number of cells. Methods 2015;72:51-6.

36. Zhou J, Sears RL, Xing X, et al. Tissue-specific DNA methylation is conserved across human, mouse, and rat, and driven by primary sequence conservation. BMC Genomics 2017;18:724. http://doi.org/10.1186/s12864-017-4115-6.

37. Head JA. Patterns of DNA methylation in animals: an ecotoxicological perspective. Integr Comp Biol 2014;54:77-86.

38. Keller U. Nutritional laboratory markers in malnutrition. J Clin Med 2019;8:775. https://doi.org/10.3390/jcm8060775.

39. Parens R, Nijhout HF, Morales A, Costa FX, Bar-Yam Y. A possible link between pyriproxyfen and microcephaly. PLoS Curr 2017;9:ecurrents.outbreaks.5afb0bfb8cf31d9a4baba7b19b4edbac. https://doi.org/10.1371/currents.outbreaks.5afb0bfb8cf31d9a4baba7b19b4edbac

40. Audouze K, Taboureau O, Grandjean P. A systems biology approach to predictive developmental neurotoxicity of a larvicide used in the prevention of Zika virus transmission. Toxicol Appl Pharmacol 2018;354:56-63.

41. Chiavegato Filho ADP. Zika realmente causa microcefalia? Folha de São Paulo. March 1, 2016. 